\title{
Analysis of Radiation-Pressure Induced Mechanical Oscillation of an Optical Microcavity
}

\author{
T. J. Kippenberg, H. Rokhsari, T. Carmon, A. Scherer, and K. J. Vahala* \\ Thomas J. Watson Laboratory of Applied Physics, California Institute of Technology, Pasadena, California 91125, USA
}

(Received 14 December 2004; published 12 July 2005)

\begin{abstract}
The theoretical work of Braginsky predicted that radiation pressure can couple the mechanical, mirror eigenmodes of a Fabry-Pérot resonator to its optical modes, leading to a parametric oscillation instability. This regime is characterized by regenerative mechanical oscillation of the mechanical mirror eigenmodes. We have recently observed the excitation of mechanical modes in an ultrahigh $Q$ optical microcavity. Here, we present a detailed experimental analysis of this effect and demonstrate that radiation pressure is the excitation mechanism of the observed mechanical oscillations.
\end{abstract}

DOI: 10.1103/PhysRevLett.95.033901

The work of Braginsky [1,2] predicted that pressure induced by circulating radiation in a Fabry-Pérot resonator can couple the optical modes to the mechanical mirror eigenmodes. The coupling can lead to a parametric oscillation instability, characterized by regenerative oscillation of the mechanical mirror eigenmodes. This mechanism has been studied theoretically for its possible role in setting a detection sensitivity limit in the Laser Interferometer Gravitational Wave Observatory (LIGO) [3,4], but has so far not been observed experimentally.

Recently, we have observed a nonlinear mechanism [5] in ultrahigh $Q$ toroid microcavities [6] that is distinct from other nonlinear mechanisms already observed in these structures $[7,8]$. The geometry for observation of this nonlinearity is a standard one in which an optical wave (here referred to as the pump) is coupled from a waveguide to a microcavity mode. The nonlinearity manifests itself as oscillations in the pump power transmitted past the microcavity. These oscillations are observed to occur at a distinct pump threshold and have spectral components at characteristic frequencies. Numerical modeling and spectral analysis reported in Ref. [5] revealed that the observed oscillations are due to regenerative oscillation of certain mechanical eigenmodes of the toroid microcavity. In this Letter, we demonstrate that the observed mechanical oscillations are caused by radiation pressure, and specifically rule out another mechanism (thermal effects [9]). As such, this work confirms the first observation [5] of radiation-pressure-induced parametric oscillation instability.

The theoretical treatment of Braginsky [1] considered mechanical oscillations of Fabry-Pérot mirror eigenmodes which lead to Stokes and anti-Stokes sidebands (at frequencies, $\omega_{0} \pm \omega_{m}$ where $\omega_{0}$ is the optical and $\omega_{m}$ the mechanical frequency). It was shown [1] that if the Stokes field coincides with an adjacent optical cavity mode the phenomenon of parametric oscillation instability can occur. In contrast to the Braginsky theory, we observed mechanical oscillations of several mechanical modes (above a certain threshold) when the mechanical resonance frequencies $\left(\omega_{m}\right)$ produce Stokes and anti-Stokes fields that fall within the same cavity resonance (i.e., $\omega_{m}<\frac{\omega_{0}}{Q}$ )
PACS numbers: 42.65.Sf, 42.65.Ky, 42.65.Yj

[5]. For $Q$ factors in the range of $10^{6}-10^{8}$ this corresponds to frequencies in the range of ca. $1-100 \mathrm{MHz}$, which coincides with the range of the first three fundamental mechanical modes of the toroid microcavities employed in this work. Figure 1(a) shows the first three mechanical modes of a toroid microcavity and Fig. 1(b) their frequency dependence on cavity length. Note that the mechanical motion causes modulation of the optical path length of the toroid cavity modes, causing the excitation of sidebands. These fields appear in the cavity transmission spectrum, as shown in Fig. 1(c).

To account for this scenario we have extended the coupled-mode analysis of Braginsky to the present case of Stokes and anti-Stokes frequency pairs falling within the same cavity resonance (for simplicity only one pair is considered here). In addition, optical coupling effects associated with the waveguide-resonator junction are, by necessity, included in the analysis. Using the rotating wave and the slowly varying envelope approximation for all field amplitudes, the mutual coupling of the pump $\left(a_{\mathrm{P}}\right)$, Stokes $\left(a_{\mathrm{S}}\right)$, anti-Stokes $\left(a_{\mathrm{AS}}\right)$, and mechanical mode $\left(x_{m}\right)$ can be described by the following coupled-mode equations:

$$
\begin{aligned}
& \frac{\partial x_{m}}{\partial t}=-\frac{1}{2 \tau_{m}} x_{m}+\frac{-i K_{o m}}{2 \sqrt{m_{\mathrm{eff}}} C(\Gamma)}\left(a_{\mathrm{P}}^{*} a_{\mathrm{AS}}+a_{\mathrm{P}} a_{\mathrm{S}}^{*}\right), \\
& \frac{\partial a_{\mathrm{P}}}{\partial t}=-\frac{a_{\mathrm{P}}}{2 \tau}+i \Delta \omega a_{\mathrm{P}}+\frac{i K_{m o}}{\sqrt{m_{\mathrm{eff}}} \omega_{m}}\left(x_{m}^{*} a_{\mathrm{AS}}+x_{m} a_{\mathrm{S}}\right)+\kappa s, \\
& \frac{\partial a_{\mathrm{S}}^{*}}{\partial t}=-\frac{1}{2 \tau} a_{\mathrm{S}}^{*}-i\left(\Delta \omega-\omega_{m}\right) a_{\mathrm{S}}^{*}-\frac{i K_{m o}}{\sqrt{m_{\mathrm{eff}}} \omega_{m}} x_{m} a_{\mathrm{P}}^{*}, \\
& \frac{\partial a_{\mathrm{AS}}}{\partial t}=-\frac{1}{2 \tau} a_{\mathrm{AS}}+i\left(\Delta \omega+\omega_{m}\right) a_{\mathrm{AS}}+\frac{i K_{m o}}{\sqrt{m_{\mathrm{eff}}} \omega_{m}} x_{m} a_{\mathrm{P}} .
\end{aligned}
$$

In these equations, the optical pump is detuned from the cavity-mode line center by $\Delta \omega=\omega-\omega_{0}$. The Stokes and anti-Stokes frequencies lie within the resonance bandwidth of the pump mode, and, correspondingly, are detuned by $\Delta \omega_{\mathrm{AS}}=\left(\Delta \omega+\omega_{m}\right)$ and $\Delta \omega_{\mathrm{S}}=\left(\Delta \omega-\omega_{m}\right)$. The first equation describes the mechanical eigenmode where $\left|x_{m}\right|^{2}$ is normalized to mechanical energy, i.e., 


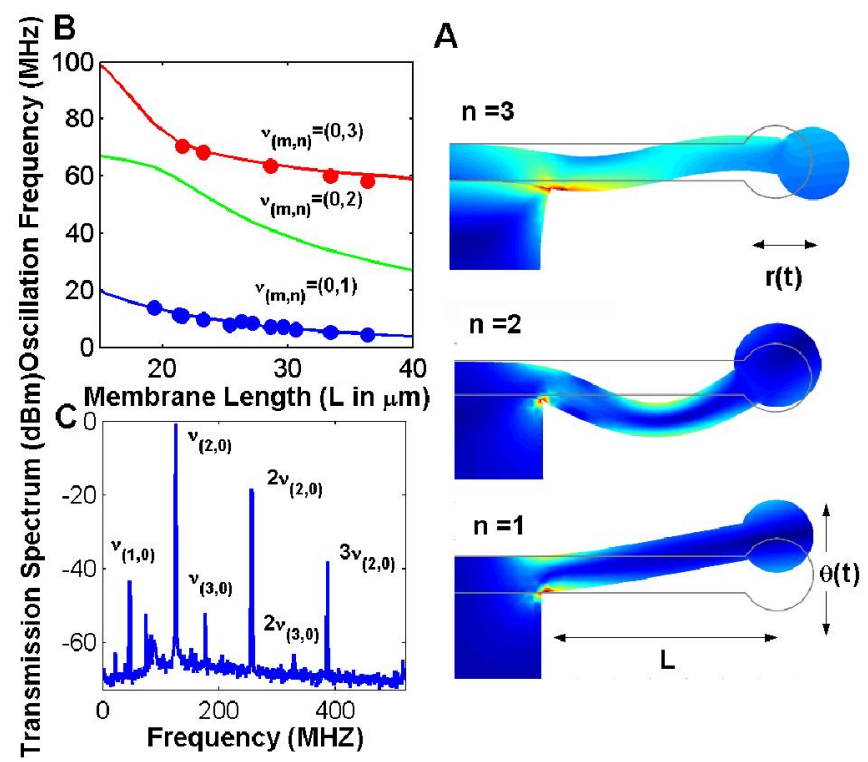

FIG. 1 (color online). (a) Finite element modeling of the micromechanical resonances of a silica toroid microcavity (diameter of the toroid is $4 \mu \mathrm{m}$ ). The radial and azimuthal mode order are denoted with $n$ and $m$ (where $m=0$ corresponds to rotationally symmetric modes). Shown are the first three rotationally symmetric radial modes $(n=1,2,3, m=0)$ in cross section with the amplitude of motion greatly exaggerated for clarity. In addition, the stress field is indicated using color. Note that the mechanical motion modulates the cavity path length due to a change in the cavity radius, which causes the excitation of optical sidebands. (b) Mechanical oscillation frequency versus the cavity length $L$ for the first three fundamental mechanical modes (dots are experimentally measured frequencies from Ref. [5]). (c) Typical frequency spectrum of the optical cavity transmission, revealing the presence of several sidebands that correspond to the first three mechanical eigenmodes $\left(\omega_{m}\right)$, as well as harmonics of $\omega_{m}$. Here, only the $n=2$ mode is above threshold, whereas the remaining mechanical modes are subthreshold (and observable due to their room temperature thermal displacement noise).

$E=\Sigma_{i} \int \epsilon_{i} \sigma_{i} d V$ (where $\epsilon_{i}$ and $\sigma_{i}$ are the diagonal components of the strain and stress tensor) which decays with the lifetime $\tau_{m}\left(Q_{m}=\omega_{m} \tau_{m}\right)$. Correspondingly, $\left|a_{\mathrm{P}}\right|^{2}$ is the energy in the pump mode, $|s|^{2}$ is the launched pump power in the waveguide. The total lifetime of the optical modes is given by $\frac{1}{\tau}=\frac{1}{\tau_{0}}+\frac{1}{\tau_{\mathrm{ex}}}$, where the external lifetime $\left(\tau_{\mathrm{ex}}\right)$ describes coupling of the microcavity mode to the waveguide via $\kappa=i \sqrt{\frac{1}{\tau_{\mathrm{ex}}}}$ and $K \equiv \frac{\tau_{0}}{\tau_{\mathrm{ex}}}$ is the normalized coupling constant. $C(\Gamma)$ is a correction factor $[1, \ldots, 2]$ due to the reduction of circulating power in the presence of modal coupling [10]. $K_{m o} \equiv \frac{\omega_{0}}{R}$ describes the mechanically induced displacement of the optical cavity resonant frequency and contains, in general, a contribution from direct spatial change as well as refractive index changes (stressoptical effect) $[11,12]$. The effective coupling of the optical mode to the mechanical mode is governed by $K_{o m} \equiv \frac{1}{R n_{\text {eff }}}$ in the case of radiation pressure [1]. The effective mass $m_{\text {eff }}$ appearing in Eq. (1) is calculated numerically by evaluating the total mechanical energy $E_{m}$ in the mechanical mode and the corresponding harmonic radial displacement (amplitude $r$ ) of the toroid periphery wherein the optical mode circulates [13]. Solving the coupled-mode equations in steady state, the pump-power threshold for the onset of mechanical oscillations is given by

$$
\begin{aligned}
P_{\text {thresh }}= & \left(\frac{\omega_{m}}{\omega_{0}}\right) R^{2} m_{\mathrm{eff}} \frac{1}{\tau_{m}} \frac{1}{\tau_{0}} \frac{\left|1+K+2 i \Delta \omega \tau_{0}\right|^{2}}{4 K} \frac{1}{2 \tau} \\
& \times\left[\frac{1}{1+4 \tau^{2} \Delta \omega_{\mathrm{AS}}^{2}}-\frac{1}{1+4 \tau^{2} \Delta \omega_{\mathrm{S}}^{2}}\right]^{-1} .
\end{aligned}
$$

Careful inspection of the last term of the threshold equation shows that mechanical gain is possible (i.e., positive threshold power) for $\omega>\omega_{0}$ (i.e., $\Delta \omega>0$ ). For $\Delta \omega<$ 0 , the mechanical mode is cooled. The need to overcome mechanical loss leads to the $\frac{1}{\tau_{m}}$ dependence, while the dependence of radiation pressure upon circulating optical power leads to the $\frac{1}{\tau_{0}}$ dependence as well as the presence of a weighting factor describing the effect of waveguide coupling $K \equiv \tau_{0} / \tau_{\mathrm{ex}}$ and pump detuning $\Delta \omega$. The optical- $Q$ scaling dependences fall into two regimes. The first occurs when $\omega_{m}<\frac{1}{\tau}$. In this regime the mechanical oscillation threshold exhibits an inverse cubic dependence on optical $Q\left[P \propto \frac{1}{Q_{m}}\left(\frac{1}{Q_{0}}\right)^{3}\right]$. In contrast, for $\omega_{m}>\frac{1}{\tau}$ [herein called the high-frequency (HF) regime], the rapid $1 / Q_{0}^{3}$ dependence is reduced because the Stokes field buildup is less and less effective in creating radiation pressure. In this regime, minimum threshold can be shown to occur overcoupled (i.e., $K>1$ ), where again the condition $\omega_{m}<\frac{1}{\tau}$ is met (i.e., the mechanical oscillation frequency is again less than the "loaded" cavity bandwidth), which causes the minimum threshold (i.e., $\frac{\partial^{2} P}{\partial K \partial \Delta \omega}=0$ [14]) to approach an asymptotic value. The transition to the HF regime, under conditions of optimum threshold, occurs for an optical $Q$ given by $Q_{0}^{\mathrm{HF}} \approx \frac{\omega_{0}}{\omega_{m}}$.

To confirm these theoretical predictions the threshold dependences [as given by Eq. (2)] on both optical and mechanical $Q$ factors have been measured. The data presented are taken using a single microtoroid device. Coupling to the resonator was performed using a fiberoptic taper coupler (see inset of Fig. 2). The microtoroid under consideration had principal, pillar, and minor diameters of 72,36 , and $6.8 \mu \mathrm{m}$, respectively, and possessed mechanical resonances frequencies at 4.4, 25.6, and $49.8 \mathrm{MHz}$ for the first three mechanical modes $(n=1,2$, 3 , and $m=0$ ). The optical pump wavelength was $\sim 1550 \mathrm{~nm}$ and mechanical oscillation instability was observed by detecting the characteristic oscillations [15] in the transmitted pump power [compare Fig. 1(c)] [5] using an electrical spectrum analyzer as described in Ref. [5]. Optimization of coupling $(K)$ was performed by adjustment of the gap between the fiber taper and the microtoroid as described in Refs. [7,8]. To measure the dependence of 
the oscillation threshold on $Q_{m}$, a silica microprobe was brought into contact with the interior (disk region) of the toroid structure. Variation of the probe contact force thereby modified mechanical $Q$ while leaving the optical $Q$ unaffected. The microprobe, which was made from an optical fiber, had a tip diameter of $\sim 2 \mu \mathrm{m}$ and can be seen in the inset of Fig. 2. In the absence of probe contact, $Q_{m}$ was measured to be $\sim 5000$ for the $n=1$ mode, and upon a progressive increase in tip pressure could be continuously decreased to $Q_{m} \approx 50$. Below threshold, the thermal displacement of the mechanical eigenmodes (the temperature being $300 \mathrm{~K}$ ) provides sufficient modulation to be optically detectable, causing the appearance of Lorentzian peaks in the cavity transmission spectrum. $Q_{m}$ was then determined by fitting the transmission spectrum with a Lorentzian, as shown in the inset of Fig. 2. For each $Q_{m}$, the minimum threshold was measured for the $n=1$ flexural mode as shown in Fig. 2. The solid line in the main panel shows that the data exhibit the $1 / Q_{m}$ dependence in agreement with Eq. (2), and Ref. [1].

We next measured the threshold dependence on the optical $Q$ factor as shown in Fig. 3 for both the $n=1$ (main panel) and the $n=3$ (inset) mechanical modes. The optical $Q$ factor was adjusted by exciting different radial and transverse optical modes. For lower optical $Q$, wherein the acoustical oscillation frequency falls within the cavity bandwidth, the rapid $1 / Q^{3}$ dependence is observed for $n=$ 1 as predicted. For higher optical $Q$, as theoretically predicted, a transition into the $\mathrm{HF}$ regime occurs at $Q_{0}^{\mathrm{HF}} \approx$ $10^{7}$. This point agrees well with the theoretical prediction $\left(\frac{\omega_{0}}{\omega_{m}}\right)$. It is important to note that these observations rule out thermal effects [9] as the origin of the observed oscillations

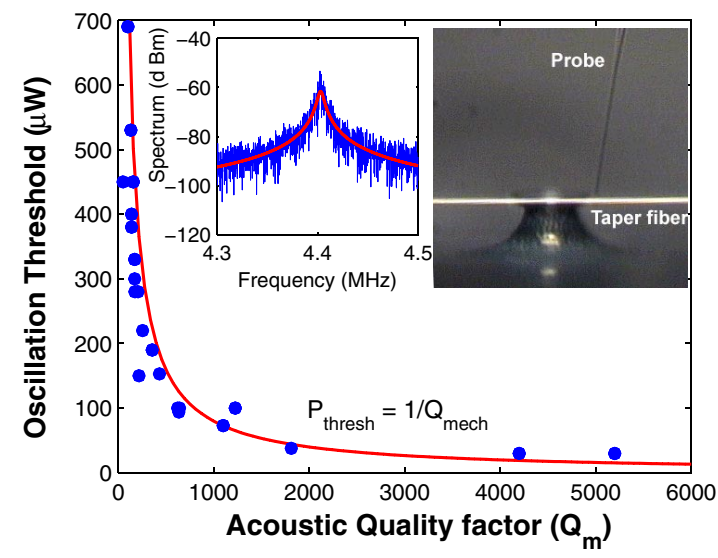

FIG. 2 (color online). Main panel: The oscillation threshold ( $\mu$ Watts) versus the mechanical quality factor of the $n=1$ mode. The solid line is the theoretical prediction based on an inverse $Q$ relation $\left(P \propto 1 / Q_{m}\right)$. Right inset: Side-view, optical micrograph of the experimental setup, consisting of a silica microprobe in contact with a taper-fiber-coupled microtoroid of $72 \mu \mathrm{m}$-principal diameter. Left inset: Spectrum of the optical cavity transmission exhibiting the thermal displacement noise of the $n=1$ mechanical mode. Solid line: The Lorentzian fit to infer the value of $Q_{m}$.
[16]. In Fig. 3, the solid line is the minimum threshold, i.e., $\left(\frac{\partial^{2} P}{\partial K \partial \Delta \omega}=0\right)$ as given by Eq. (2). With the exception of the effective mass, $m_{\text {eff }}$, all parameters used to create this plot were experimentally measured parameters [i.e., $C(\Gamma), R$, $\left.Q_{m}, \omega_{m}, Q_{0}, \omega_{0}\right]$. The effective mass $m_{\text {eff }}$ was inferred to be $m_{\mathrm{eff}}^{(1)}=3.3 \times 10^{-8} \mathrm{~kg}$.

The inset of Fig. 3 shows the measured threshold versus optical $Q$ for the $n=3$ mode. The $n=3$ mode threshold dependence shows that this mode is already well into the HF regime, exhibiting the theoretically predicted asymptotic behavior of the minimum threshold. This fact is consistent with the observed resonance frequency, $49 \mathrm{MHz}$, for the $n=3$ mode which predicts that the HF regime occurs for optical $Q$ factors in excess of $10^{7}$ $\left(Q_{0}^{\mathrm{HF}}=\frac{\omega_{0}}{\omega_{m}}=3.8 \times 10^{6}\right)$. Comparison with the $n=1$ mode data shows that oscillation on the $n=3$ mode is preferred for lower optical $Q$ s. Indeed, preference to the $n=3$ mode was possible by loading the microcavity into the overcoupled regime, in agreement with theory. The solid curve in the inset gives the single-parameter fit to the $n=3$ data which yields $m_{\mathrm{eff}}^{(3)}=5 \times 10^{-11} \mathrm{~kg}$, which is a factor of 660 lower than the mass of the $n=1$ mode.

As a further test of the validity of the theoretical model, the experimental effective mass values are compared with the theoretical prediction based on finite element modeling. For the $n=3$ mode, the predicted mass associated with the radial motion was $m_{\text {eff }}^{(3)}=5 \times 10^{-11} \mathrm{~kg}$, which is in very good agreement with the experimental fit. However, for the $n=1,2$ modes, the calculated effective mass is a

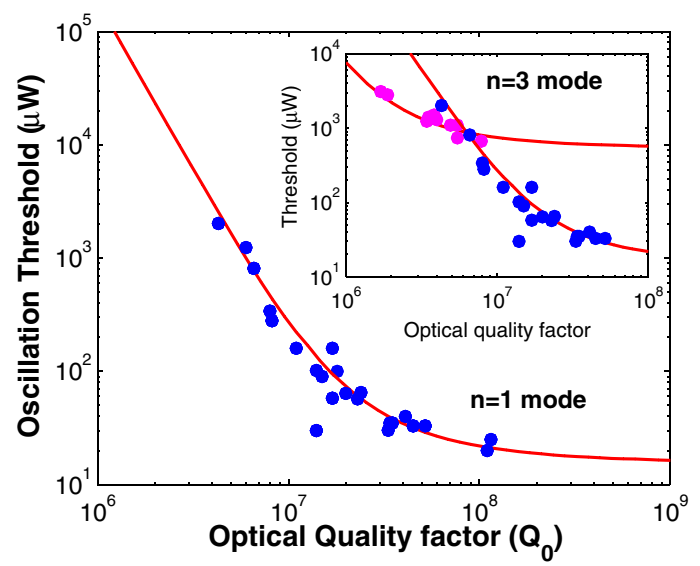

FIG. 3 (color online). Main panel: The measured mechanical oscillation threshold ( $\mu$ Watts) plotted versus the optical $Q$ factor for the fundamental flexural mode $\left(n=1, \nu_{m}=4.4 \mathrm{MHz}\right.$, $Q_{m}=3500$ ). The solid line is a one-parameter theoretical fit obtained from the minimum threshold equation by first performing a minimization with respect to coupling and pump wavelength detuning, and then fitting by adjustment of the effective mass $\left(m_{\text {eff }}^{(1)}=3.3 \times 10^{-8} \mathrm{~kg}\right)$. Inset: The measured threshold for the 3rd order mode $\left(n=3, \nu_{m}=49 \mathrm{MHz}, Q=2500\right)$ plotted versus optical $Q$. The solid line gives again the theoretical prediction with $m_{\text {eff }}^{(3)}=0.5 \times 10^{-10} \mathrm{~kg}$. The $n=1$ mode data from the main panel is superimposed for comparison. 


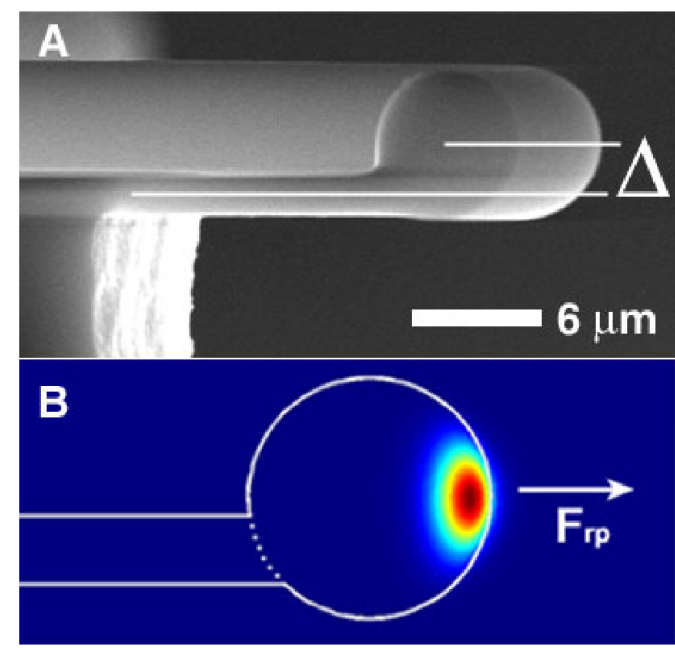

FIG. 4 (color online). (a) Scanning-electron micrograph of the microcavity cross section achieved using focused-ion beam (FIB) preparation. The image reveals the presence of an offset in the toroid with respect to the $2 \mu \mathrm{m}$ thick silica support disk (offset of ca. $1.3 \mu \mathrm{m}$ ). (b) Finite element modeling of the fundamental optical mode. The presence of an offset provides a moment arm that effectively enhances coupling of radiation pressure to the $n=1$ transverse motion.

strong function of the offset of the toroidal ring with respect to the equatorial plane of the disk [17]. To both validate and quantify this offset, a cross section of the toroid microcavity used in this study was obtained with focused-ion beam slicing. Scanning electron microscopy imaging [cf. Fig. 4(a)] reveals the presence of the abovepostulated equatorial offset which amounts to $\Delta=$ $1.3 \mu \mathrm{m}$. Incorporation of this offset into the numerical mass calculation yields $m_{\mathrm{eff}}^{(1)}=2.6 \times 10^{-8} \mathrm{~kg}$ and $m_{\mathrm{eff}}^{(2)}=$ $2 \times 10^{-9} \mathrm{~kg}$. This value agrees very well with the experimental values from above. Finally, the numerical model also explains why the $n=2$ mode is observed only subthreshold in the experiments. The low mechanical $Q$ value $(\sim 200)$ in conjunction with its high effective mass and frequency, predicts threshold powers $>2 \mathrm{~mW}$, which are higher than pump powers available in the experiments.

In summary, presented is both an experimental and a theoretical analysis of radiation-pressure-induced parametric oscillation instability, as predicted by Braginsky. Excellent agreement of the threshold functional dependence on optical $Q$ is obtained, providing a confirmation that radiation pressure is the excitation mechanism of the observed oscillations. Besides the fundamental aspects of this work, the observed coupling of mechanical and optical modes by radiation pressure can find applications in micromechanical and nanomechanical systems [18] for ultrahigh sensitivity measurements of charge [19], displacement [20], mass, force [20], or biological entities [21]. Equally important, radiation pressure as observed here can be used to achieve cooling of a mechanical resonator modes [22].

This work was supported by the NSF, DARPA, AFSOR, and the Center for the Physics of Information.
*Electronic address: vahala@its.caltech.edu

[1] V. B. Braginsky, S. E. Strigin, and S. P. Vyatchanin, Phys. Lett. A 287, 331 (2001).

[2] V. B. Braginsky, S. E. Strigin, and S. P. Vyatchanin, Phys. Lett. A 305, 111 (2002).

[3] A. Abramovici et al., Science 256, 325 (1992).

[4] G. Amelino-Camelia, Nature (London) 398, 216 (1999).

[5] H. Rokhsari, T. Kippenberg, T. Carmon, and K. Vahala (to be published).

[6] D. K. Armani, T. J. Kippenberg, S. M. Spillane, and K. J. Vahala, Nature (London) 421, 925 (2003).

[7] T. J. Kippenberg, S. M. Spillane, and K. J. Vahala, Phys. Rev. Lett. 93, 083904 (2004).

[8] S. M. Spillane, T. J. Kippenberg, and K. J. Vahala, Nature (London) 415, 621 (2002).

[9] M. Zalalutdinov, A. Zehnder, A. Olkhovets, S. Turner, L. Sekaric, B. Ilic, D. Czaplewski, J. M. Parpia, and H. G. Craighead, Appl. Phys. Lett. 79, 695 (2001).

[10] T. J. Kippenberg, S. M. Spillane, and K. J. Vahala, Opt. Lett. 27, 1669 (2002).

[11] From the simulations it is calculated that the change in refractive index due to the stress-optical effect is more than 1 order of magnitude smaller compared to the direct spatial change in cavity path length [i.e., $2 \pi(R+d R) \times$ $\left.\left(\Delta n+n_{\mathrm{eff}}\right) \approx 2 \pi n_{\mathrm{eff}}(R+d R)\right]$.

[12] R. M. Shelby, M.D. Levenson, and P.W. Bayer, Phys. Rev. B 31, 5244 (1985).

[13] Note that the mechanical energy $\left(E_{m}\right)$ is asssociated with both a radial motion $\left(\frac{1}{2} m_{\mathrm{eff}} \omega_{m} r^{2}\right)$ and a transverse motion. However, to highest order, only the radial motion modulates the cavity optical path length so that the effective mass is given by calculating numerically $m_{\text {eff }}=E_{m} /\left(\omega_{m}^{2} r^{2}\right)$

[14] Note that minimum threshold requires an optimization of $P_{\text {thresh }}$ with respect to both cavity loading $(K)$ and pump wavelength detuning $\Delta \omega$ (i.e., $\frac{\partial^{2} P}{\partial K \partial \Delta \omega}=0$ ) which is always done in the measurements.

[15] The temporal behavior has been reported in T. Carmon, $\mathrm{H}$. Rokhsari, L. Yang, T. J. Kippenberg, and K. J. Vahala, Phys. Rev. Lett. 94, 223902 (2005).

[16] Thermal instabilities are not dependent upon resonant buildup since the thermo-optic mechanical coupling is dependent upon the coupled (i.e., absorbed) power into the cavity, not the circulating power. The expected threshold dependence on optical $Q$ for thermal instabilities is therefore $\frac{1}{Q_{0}^{2}}$, which is not observed in the current experiments.

[17] We note that such an offset must exist, since mechanical motion of the $n=1,2$ modes causes modulation of the path length at the frequency $2 \omega_{m}$ (i.e., frequency doubling), which is not observed in experiments.

[18] H. G. Craighead, Science 290, 1532 (2000).

[19] A. N. Cleland and M. L. Roukes, Nature (London) 392, 160 (1998).

[20] D. Rugar, R. Budakian, H. J. Mamin, and B.W. Chui, Nature (London) 430, 329 (2004).

[21] B. Ilic, Y. Yang, and H. G. Craighead, Appl. Phys. Lett. 85, 2604 (2004).

[22] C. H. Metzger and K. I. Karrai, Nature (London) 432, 1002 (2004). 